PROCEEDINGS OF THE

AMERICAN MATHEMATICAL SOCIETY

Volume 139, Number 4, April 2011, Pages 1347-1360

S 0002-9939(2010)10817-1

Article electronically published on November 23, 2010

\title{
VECTOR SPACES OF ENTIRE FUNCTIONS OF UNBOUNDED TYPE
}

\author{
JERÓNIMO LÓPEZ - SALAZAR CODES \\ (Communicated by Mei-Chi Shaw)
}

\begin{abstract}
Let $E$ be an infinite dimensional complex Banach space. We prove the existence of an infinitely generated algebra, an infinite dimensional closed subspace and a dense subspace of entire functions on $E$ whose non-zero elements are functions of unbounded type. We also show that the $\tau_{\delta}$ topology on the space of all holomorphic functions cannot be obtained as a countable inductive limit of Fréchet spaces.
\end{abstract}

\section{INTRODUCTION AND NOTATION}

The results in this article are related to the lineability of sets of functions. We recall that a subset $X$ of a vector space is said to be lineable if there exists an infinite dimensional vector space $W$ such that $W \subset X \cup\{0\}$. The first theorems in this topic were obtained by Gurariy in the sixties (see [12]), and in the last ten years this line of research has been extensively developed by several authors such as Aron, Bernal González, García Pacheco, Seoane Sepúlveda and others, who have proved the lineability of many collections of special mappings: differentiable nowhere monotone functions, everywhere surjective functions, continuous nowhere differentiable functions, etc. (see [5], 6], 7]).

In this paper, we study the lineability of the set of unbounded holomorphic functions defined on a Banach space. To begin with, let us explain the main notation used in our work. Throughout the article, $E$ will denote a complex Banach space and $U$ will be an open subset of $E$. If $x \in E$ and $r>0$, then $B(x, r)$ represents the open ball in $E$ with center $x$ and radius $r$. For each $n \in \mathbb{N}, \mathcal{P}\left({ }^{n} E\right)$ denotes the space of all continuous $n$-homogeneous polynomials from $E$ into $\mathbb{C}$. The symbol $\mathcal{H}(U)$ denotes the space of all holomorphic functions on $U$. If $f \in \mathcal{H}(U)$ and $X$ is a subset of $U$, let

$$
\|f\|_{X}=\sup \{|f(x)|: x \in X\} .
$$

Let

$$
\mathcal{H}_{b}(E)=\left\{f \in \mathcal{H}(E):\|f\|_{B}<\infty \text { for every bounded set } B \subset E\right\} .
$$

If $E$ has infinite dimension, then $\mathcal{H}_{b}(E)$ is always a proper subset of $\mathcal{H}(E)$ (see [11, page 157]); the elements of $\mathcal{H}(E) \backslash \mathcal{H}_{b}(E)$ are called entire functions of unbounded type.

Received by the editors February 22, 2010 and, in revised form, April 7, 2010.

2010 Mathematics Subject Classification. Primary 46G20; Secondary 46E50.

Key words and phrases. Entire function, lineable set, $\tau_{\delta}$ topology.

The author was supported by UCM Grant \#BE46/08.

(C)2010 American Mathematical Society Reverts to public domain 28 years from publication 
The symbol $\tau_{\delta}$ denotes the locally convex topology on $\mathcal{H}(U)$ defined by the seminorms $p$ which verify the following property: for each increasing countable open cover $\left\{V_{n}\right\}_{n=1}^{\infty}$ of $U$, there exist $C>0$ and $n \in \mathbb{N}$ such that

$$
p(f) \leq C\|f\|_{V_{n}} \quad \text { for every } f \in \mathcal{H}(U) .
$$

$\tau_{\delta}$ is the bornological topology associated with the compact open topology $\tau_{0}$ on $\mathcal{H}(U)$; in particular, $\tau_{0} \leq \tau_{\delta}$. We refer the reader to the books of Dineen [11] and Mujica 14 for extensive studies of holomorphic functions defined on Banach spaces and the topologies on $\mathcal{H}(U)$.

\section{Lineability of $\mathcal{H}(E) \backslash \mathcal{H}_{b}(E)$}

In this section we will see how the set $\mathcal{H}(E) \backslash \mathcal{H}_{b}(E)$ is algebrable, spaceable and dense-lineable. This means that there exist an infinitely generated algebra $\mathcal{A} \subset \mathcal{H}(E)$, an infinite dimensional closed subspace $\mathcal{F} \subset\left(\mathcal{H}(E), \tau_{0}\right)$ and a dense subspace $W \subset\left(\mathcal{H}(E), \tau_{\delta}\right)$ such that

$$
\mathcal{A} \backslash\{0\} \subset \mathcal{H}(E) \backslash \mathcal{H}_{b}(E), \quad \mathcal{F} \backslash\{0\} \subset \mathcal{H}(E) \backslash \mathcal{H}_{b}(E)
$$

and

$$
W \backslash\{0\} \subset \mathcal{H}(E) \backslash \mathcal{H}_{b}(E) .
$$

To get these results we need a proposition about biorthogonal systems in Banach spaces, which will be our main tool throughout the article.

Proposition 1 (see 9]). Let $E$ be an infinite dimensional Banach space. Let $x_{0} \in E$ and $\varphi_{0} \in E^{\prime}$. Then there are sequences $\left\{x_{k}\right\}_{k=1}^{\infty} \subset E$ and $\left\{\varphi_{k}\right\}_{k=1}^{\infty} \subset E^{\prime}$ with the following properties:

a) $\lim _{k \rightarrow \infty} \varphi_{k}(x)=0$ for all $x \in E$,

b) $\left\|\varphi_{k}\right\|=1$ for all $k \geq 1$,

c) $\sup _{k \in \mathbb{N}}\left\|x_{k}\right\|<\infty$,

d) $\varphi_{k}\left(x_{k}\right)=1$ for all $k \geq 1$,

e) if $k, j \in \mathbb{N} \cup\{0\}$ and $k \neq j$, then $\varphi_{k}\left(x_{j}\right)=0$.

The proof of this proposition is the same as in [9, page 250] and it is an inductive process with the subspaces $X_{1}=\left\{x \in E: \varphi_{0}(x)=0\right\}$ and $Z_{1}=\left\{\varphi \in E^{\prime}: \varphi\left(x_{0}\right)=\right.$ $0\}$ in the first step.

Theorem 2. If $E$ is an infinite dimensional Banach space, then there exists an infinitely generated algebra $\mathcal{A} \subset \mathcal{H}(E)$ such that $\mathcal{A} \backslash\{0\} \subset \mathcal{H}(E) \backslash \mathcal{H}_{b}(E)$.

Proof. Let $\left\{x_{k}, \varphi_{k}\right\}_{k=1}^{\infty}$ be a biorthogonal system in $E$ with the properties given by Proposition 1, in this case, we do not need any particular vector $x_{0}$ or functional $\varphi_{0}$. Let $\left\{a_{j}\right\}_{j=1}^{\infty}$ be a strictly increasing sequence of prime numbers. For every $j \in \mathbb{N}$, the function

$$
f_{j}=\sum_{k=1}^{\infty}\left(a_{j} \cdot \varphi_{k}\right)^{k}
$$


is entire on $E$ (see [14, Example 5.5]). Let $\mathcal{A}$ be the algebra generated by $\left\{f_{j}\right\}_{j=1}^{\infty}$. Every $h \in \mathcal{A} \backslash\{0\}$ can be written as

$$
h=\sum_{n=1}^{N} \lambda_{n} \prod_{j \in J_{n}} f_{j}^{p_{n, j}}
$$

where $N \in \mathbb{N}, \lambda_{n} \in \mathbb{C} \backslash\{0\}, J_{n}$ is a finite subset of $\mathbb{N}$ and $p_{n, j} \in \mathbb{N}$ for all $n, j$. We assume that if $J_{n}=J_{m}$ and $p_{n, j}=p_{m, j}$ for every $j \in J_{n}=J_{m}$, then $n=m$. Let us compute the function $h$ at the points $\left\{x_{k}\right\}_{k=1}^{\infty}$ :

$$
\begin{aligned}
h\left(x_{k}\right) & =\sum_{n=1}^{N} \lambda_{n} \prod_{j \in J_{n}}\left(f_{j}\left(x_{k}\right)\right)^{p_{n, j}}=\sum_{n=1}^{N} \lambda_{n} \prod_{j \in J_{n}}\left(a_{j}^{k}\right)^{p_{n, j}} \\
& =\sum_{n=1}^{N} \lambda_{n}\left(\prod_{j \in J_{n}} a_{j}^{p_{n, j}}\right)^{k} .
\end{aligned}
$$

Since the numbers $\left\{a_{j}\right\}_{j=1}^{\infty}$ are prime, the products $\prod_{j \in J_{1}} a_{j}^{p_{1, j}}, \ldots, \prod_{j \in J_{N}} a_{j}^{p_{N, j}}$ are different, so there exists $m \in\{1, \ldots, N\}$ such that

$$
\prod_{j \in J_{m}} a_{j}^{p_{m, j}}>\prod_{j \in J_{n}} a_{j}^{p_{n, j}} \quad \text { for all } n \in\{1, \ldots, N\} \backslash\{m\} .
$$

Hence

$$
\frac{h\left(x_{k}\right)}{\left(\prod_{j \in J_{m}} a_{j}^{p_{m, j}}\right)^{k}}=\lambda_{m}+\sum_{\substack{n=1 \\ n \neq m}}^{N} \lambda_{n}\left(\frac{\prod_{j \in J_{n}} a_{j}^{p_{n, j}}}{\prod_{j \in J_{m}} a_{j}^{p_{m, j}}}\right)^{k} \underset{k \rightarrow \infty}{\rightarrow} \lambda_{m} \neq 0
$$

Moreover, each $a_{j}$ is greater than 1 , so $\lim _{k \rightarrow \infty}\left(\prod_{j \in J_{m}} a_{j}^{p_{m, j}}\right)^{k}=\infty$ and therefore $\lim _{k \rightarrow \infty}\left|h\left(x_{k}\right)\right|=\infty$. Since the sequence $\left\{x_{k}\right\}_{k=1}^{\infty}$ is bounded, it follows that $h$ is a function of unbounded type.

Finally, we will show that $\left\{f_{j}\right\}_{j=1}^{\infty}$ is an algebraically independent set. Suppose

$$
\sum_{n=1}^{N} \lambda_{n} \prod_{j \in J_{n}} f_{j}^{p_{n, j}}=0
$$

where $N \in \mathbb{N}, \lambda_{n} \in \mathbb{C}, J_{n}$ is a finite subset of $\mathbb{N}$ and $p_{n, j} \in \mathbb{N}$ for all $n, j$. If $\lambda_{n} \neq 0$ for some $n$, then it is possible to repeat the same argument used to prove that $h \in \mathcal{H}(E) \backslash \mathcal{H}_{b}(E)$ and we deduce that

$$
0=\left|\sum_{n=1}^{N} \lambda_{n} \prod_{j \in J_{n}}\left(f_{j}\left(x_{k}\right)\right)^{p_{n, j}}\right| \underset{k \rightarrow \infty}{\rightarrow} \infty .
$$

This is absurd, so $\lambda_{n}=0$ for every $n$. This proves that $\left\{f_{j}\right\}_{j=1}^{\infty}$ is an algebraically independent system of generators of $\mathcal{A}$ and this algebra is infinitely generated.

Proposition 3. Let $E$ be an infinite dimensional Banach space. Let $\left\{x_{k}, \varphi_{k}\right\}_{k=1}^{\infty}$ be a biorthogonal system in $E$ such that $\lim _{k \rightarrow \infty} \varphi_{k}(x)=0$ for all $x \in E$ and 
$R=\sup _{k}\left\|x_{k}\right\|<\infty$ (see Proposition 1). Let

$$
f=\sum_{k=1}^{\infty} \varphi_{k}^{k}
$$

If $P$ is a non-zero continuous polynomial on $E$, then $P \cdot f \in \mathcal{H}(E) \backslash \mathcal{H}_{b}(E)$.

Proof. The polynomial can be written as $P=P_{m}+\cdots+P_{0}$, where $P_{k} \in \mathcal{P}\left({ }^{k} E\right)$ for $0 \leq k \leq m$ and $P_{m} \neq 0$. Let $w \in E$ such that $\|w\|=1$ and $P_{m}(w) \neq 0$. Let $L$ be the symmetric $m$-linear function associated with $P_{m}$. The function

$$
t \in \mathbb{R} \mapsto\left|P_{m}(w)\right| t^{m}-\sum_{k=0}^{m-1}\left(\begin{array}{c}
m \\
k
\end{array}\right)\|L\| \cdot(2 R)^{m-k} \cdot t^{k}-\sum_{k=0}^{m-1}\left\|P_{k}\right\| \cdot(t+2 R)^{k}
$$

is a non-zero polynomial, and hence there exists $t>0$ such that

$$
\left|P_{m}(w)\right| t^{m}-\sum_{k=0}^{m-1}\left(\begin{array}{c}
m \\
k
\end{array}\right)\|L\| \cdot(2 R)^{m-k} \cdot t^{k}-\sum_{k=0}^{m-1}\left\|P_{k}\right\| \cdot(t+2 R)^{k} \geq\left|P_{m}(w)\right| .
$$

We define $z=t w$. Assume that $x \in E$ and $\|x-z\| \leq 2 R$. By the Newton Binomial Formula, we have

$$
P_{m}(x)=P_{m}(z)+\sum_{k=0}^{m-1}\left(\begin{array}{c}
m \\
k
\end{array}\right) L\left(z^{k},(x-z)^{m-k}\right)
$$

(see [11, Lemma 1.9]). Then

$$
\begin{aligned}
\left|P_{m}(x)\right| & \geq\left|P_{m}(z)\right|-\sum_{k=0}^{m-1}\left(\begin{array}{c}
m \\
k
\end{array}\right)\|L\| \cdot\|z\|^{k} \cdot\|x-z\|^{m-k} \\
& \geq\left|P_{m}(w)\right| t^{m}-\sum_{k=0}^{m-1}\left(\begin{array}{c}
m \\
k
\end{array}\right)\|L\| \cdot t^{k} \cdot(2 R)^{m-k} .
\end{aligned}
$$

On the other hand, since $\|x\| \leq\|z\|+2 R=t+2 R$,

$$
\sum_{k=0}^{m-1}\left|P_{k}(x)\right| \leq \sum_{k=0}^{m-1}\left\|P_{k}\right\| \cdot\|x\|^{k} \leq \sum_{k=0}^{m-1}\left\|P_{k}\right\| \cdot(t+2 R)^{k} .
$$

Therefore,

$$
\begin{aligned}
|P(x)| & \geq\left|P_{m}(x)\right|-\sum_{k=0}^{m-1}\left|P_{k}(x)\right| \\
& \geq\left|P_{m}(w)\right| t^{m}-\sum_{k=0}^{m-1}\left(\begin{array}{c}
m \\
k
\end{array}\right)\|L\| \cdot t^{k} \cdot(2 R)^{m-k}-\sum_{k=0}^{m-1}\left\|P_{k}\right\| \cdot(t+2 R)^{k} .
\end{aligned}
$$

By (2.1), we deduce that $|P(x)| \geq\left|P_{m}(w)\right|>0$ for all $x \in \bar{B}(z, 2 R)$.

The function $f$ is entire on $E$ because $\lim _{k \rightarrow \infty} \varphi_{k}(x)=0$ for all $x \in E$ (see 14, Example 5.5]). Let $k_{0} \in \mathbb{N}$ such that $\left|\varphi_{k}(z)\right|<\frac{1}{2}$ for all $k \geq k_{0}$. Since

$$
f\left(2 x_{k}+z\right)=\left(2+\varphi_{k}(z)\right)^{k}+\sum_{j=1, j \neq k}^{\infty}\left(\varphi_{j}(z)\right)^{j}
$$


if $k \geq k_{0}$, then

$$
\begin{aligned}
\left|f\left(2 x_{k}+z\right)\right| & \geq\left|2+\varphi_{k}(z)\right|^{k}-\sum_{j=1, j \neq k}^{\infty}\left|\varphi_{j}(z)\right|^{j} \\
& \geq\left(2-\left|\varphi_{k}(z)\right|\right)^{k}-\sum_{j=1}^{k_{0}}\left|\varphi_{j}(z)\right|^{j}-\sum_{j=k_{0}+1}^{\infty} \frac{1}{2^{j}} \\
& \geq\left(\frac{3}{2}\right)^{k}-\sum_{j=1}^{k_{0}}\left|\varphi_{j}(z)\right|^{j}-1 .
\end{aligned}
$$

This implies that $\lim _{k \rightarrow \infty}\left|f\left(2 x_{k}+z\right)\right|=\infty$. Since $2 x_{k}+z \in \bar{B}(z, 2 R)$ for every $k$,

$$
\lim _{k \rightarrow \infty}\left|(P \cdot f)\left(2 x_{k}+z\right)\right| \geq\left|P_{m}(w)\right| \cdot \lim _{k \rightarrow \infty}\left|f\left(2 x_{k}+z\right)\right|=\infty,
$$

so $P \cdot f \in \mathcal{H}(E) \backslash \mathcal{H}_{b}(E)$.

Theorem 4. If $E$ is an infinite dimensional Banach space, then there exists an infinite dimensional closed subspace $\mathcal{F} \subset\left(\mathcal{H}(E), \tau_{0}\right)$ such that $\mathcal{F} \backslash\{0\} \subset \mathcal{H}(E) \backslash$ $\mathcal{H}_{b}(E)$.

Proof. Let $\left\{x_{k}, \varphi_{k}\right\}_{k=1}^{\infty}$ be a biorthogonal system in $E$ with the properties given in Proposition 1, Let

$$
f=\sum_{k=1}^{\infty} \varphi_{k}^{k} \in \mathcal{H}(E)
$$

By Proposition 3 ,

$$
\mathcal{F}=\left\{\psi \cdot f: \psi \in E^{\prime}\right\}
$$

is a subspace of $\mathcal{H}(E)$ such that $\mathcal{F} \backslash\{0\} \subset \mathcal{H}(E) \backslash \mathcal{H}_{b}(E)$.

Let us suppose that $m \in \mathbb{N}, \lambda_{1}, \ldots, \lambda_{m} \in \mathbb{C}$ and

$$
\lambda_{1} \varphi_{1} \cdot f+\cdots+\lambda_{m} \varphi_{m} \cdot f=0 .
$$

If this sum is computed at the points $\left\{x_{1}, \ldots, x_{m}\right\}$, it follows that $\lambda_{1}=\cdots=\lambda_{m}=$ 0 . Therefore, $\left\{\varphi_{k} \cdot f\right\}_{k=1}^{\infty}$ is a linearly independent set and $\mathcal{F}$ has infinite dimension. Suppose $\left(\psi_{i} \cdot f\right)_{i}$ is a net in $\mathcal{F}$ which converges to a function $h \in \mathcal{H}(E)$ for the $\tau_{0}$ topology:

$$
h=\lim _{i}\left(\psi_{i} \cdot f\right)=\lim _{i} \sum_{k=1}^{\infty}\left(\psi_{i} \cdot \varphi_{k}^{k}\right) .
$$

The mapping

$$
g=\sum_{n=0}^{\infty} \frac{\widehat{d^{n}} g(0)}{n !} \in\left(\mathcal{H}(E), \tau_{0}\right) \mapsto \frac{\widehat{d^{2}} g(0)}{2} \in\left(\mathcal{P}\left({ }^{2} E\right), \tau_{0}\right)
$$

is continuous (see [11, Proposition 3.22]), so

$$
\frac{\widehat{d^{2}} h(0)}{2}=\lim _{i}\left(\psi_{i} \cdot \varphi_{1}\right)
$$

where the convergence is uniform on the compact subsets of $E$.

Let $K$ be a compact subset of $E$. Let

$$
L=\left\{x+e^{i \theta} y: x, y \in K \cup\left\{x_{1}\right\}, 0 \leq \theta \leq 2 \pi\right\},
$$


which is also a compact subset of $E$. Let $x, y \in K \cup\left\{x_{1}\right\}$. As is shown in [8, Lemma 1],

$$
\left(\psi_{i}-\psi_{j}\right)(x) \cdot \overline{\varphi_{1}(y)}=\frac{1}{2 \pi} \int_{0}^{2 \pi} e^{i \theta} \cdot\left(\psi_{i}-\psi_{j}\right)\left(x+e^{i \theta} y\right) \cdot \overline{\varphi_{1}\left(x+e^{i \theta} y\right)} d \theta
$$

Then

$$
\begin{aligned}
\left|\left(\psi_{i}-\psi_{j}\right)(x)\right| \cdot\left|\varphi_{1}(y)\right| & \leq \frac{1}{2 \pi} \int_{0}^{2 \pi}\left|\left(\psi_{i}-\psi_{j}\right)\left(x+e^{i \theta} y\right) \cdot \varphi_{1}\left(x+e^{i \theta} y\right)\right| d \theta \\
& \leq \frac{1}{2 \pi} \int_{0}^{2 \pi}\left\|\left(\psi_{i}-\psi_{j}\right) \cdot \varphi_{1}\right\|_{L} d \theta \\
& =\left\|\left(\psi_{i}-\psi_{j}\right) \cdot \varphi_{1}\right\|_{L} .
\end{aligned}
$$

Therefore,

$$
\left\|\psi_{i}-\psi_{j}\right\|_{K \cup\left\{x_{1}\right\}} \cdot\left\|\varphi_{1}\right\|_{K \cup\left\{x_{1}\right\}} \leq\left\|\left(\psi_{i}-\psi_{j}\right) \cdot \varphi_{1}\right\|_{L} .
$$

Moreover, $\left\|\varphi_{1}\right\|_{K \cup\left\{x_{1}\right\}} \geq 1$, so

$$
\left\|\psi_{i}-\psi_{j}\right\|_{K} \leq\left\|\psi_{i}-\psi_{j}\right\|_{K \cup\left\{x_{1}\right\}} \cdot\left\|\varphi_{1}\right\|_{K \cup\left\{x_{1}\right\}} \leq\left\|\left(\psi_{i}-\psi_{j}\right) \cdot \varphi_{1}\right\|_{L} .
$$

By (2.2), $\left(\psi_{i} \cdot \varphi_{1}\right)_{i}$ is a Cauchy net in $\left(\mathcal{P}\left({ }^{2} E\right), \tau_{0}\right)$, so for every $\varepsilon>0$ there is an index $i_{0}$ such that

$$
\left\|\left(\psi_{i}-\psi_{j}\right) \cdot \varphi_{1}\right\|_{L} \leq \varepsilon
$$

for $i, j \geq i_{0}$. Thus,

$$
\left\|\psi_{i}-\psi_{j}\right\|_{K} \leq\left\|\left(\psi_{i}-\psi_{j}\right) \cdot \varphi_{1}\right\|_{L} \leq \varepsilon
$$

for all $i, j \geq i_{0}$. This shows that $\left(\psi_{i}\right)_{i}$ is a Cauchy net in $\left(E^{\prime}, \tau_{0}\right)$, and hence there exists $\psi=\lim _{i} \psi_{i} \in E^{\prime}$ (see [14, Proposition 9.13]). Therefore,

$$
h=\lim _{i}\left(\psi_{i} \cdot f\right)=\psi \cdot f \in \mathcal{F}
$$

and $\mathcal{F}$ is a closed subspace in $\left(\mathcal{H}(E), \tau_{0}\right)$.

Theorem 5. If $E$ is an infinite dimensional Banach space, then there exists a dense subspace $W \subset\left(\mathcal{H}(E), \tau_{\delta}\right)$ such that $W \backslash\{0\} \subset \mathcal{H}(E) \backslash \mathcal{H}_{b}(E)$.

Proof. If $A$ is a set, then $|A|$ denotes the cardinality of $A$. For each $m \in \mathbb{N}$, let $\mathcal{B}_{m}=\left\{P_{m, i}: i \in I_{m}\right\}$ be an algebraic basis of $\mathcal{P}\left({ }^{m} E\right)$ such that $\left\|P_{m, i}\right\|=1$ for all $i$. The space

$$
\bigoplus_{n=0}^{m} \mathcal{P}\left({ }^{n} E\right)=\left\{\sum_{n=0}^{m} P_{n}: P_{0} \in \mathcal{P}\left({ }^{0} E\right), \ldots, P_{m} \in \mathcal{P}\left({ }^{m} E\right)\right\}
$$

has the same cardinality as $\mathcal{B}_{m}$. Indeed, if $n \leq m$, then $\mathcal{P}\left({ }^{n} E\right)$ is a complemented subspace of $\mathcal{P}\left({ }^{m} E\right)$ (see [4, Proposition 5.3]). Hence $\left|\mathcal{P}\left({ }^{n} E\right)\right| \leq\left|\mathcal{P}\left({ }^{m} E\right)\right|$, which implies that

$$
\begin{aligned}
\left|\mathcal{B}_{m}\right| & \leq\left|\bigoplus_{n=0}^{m} \mathcal{P}\left({ }^{n} E\right)\right|=\left|\mathcal{P}\left({ }^{0} E\right) \times \cdots \times \mathcal{P}\left({ }^{m} E\right)\right| \\
& \leq\left|\mathcal{P}\left({ }^{m} E\right)^{m+1}\right|=\left|\mathcal{P}\left({ }^{m} E\right)\right|
\end{aligned}
$$


Moreover, $\left|\mathcal{P}\left({ }^{m} E\right)\right|=\left|\mathcal{B}_{m}\right|$ because $\mathcal{P}\left({ }^{m} E\right)$ is an infinite dimensional Banach space (see [13]) and hence $\left|\bigoplus_{n=0}^{m} \mathcal{P}\left({ }^{n} E\right)\right|=\left|\mathcal{B}_{m}\right|$. Therefore, $\bigoplus_{n=0}^{m} \mathcal{P}\left({ }^{n} E\right)$ can be indexed in the following way:

$$
\bigoplus_{n=0}^{m} \mathcal{P}\left({ }^{n} E\right)=\left\{Q_{m, i}: i \in I_{m}\right\}
$$

We define

$$
W=\operatorname{span}\left\{P_{m, i} \cdot f+Q_{m, i}: m \in \mathbb{N}, i \in I_{m}\right\},
$$

where $f \in \mathcal{H}(E)$ is the function introduced in Proposition 3, Let

$$
\sum_{k=1}^{n} \lambda_{k}\left(P_{m_{k}, i_{k}} \cdot f+Q_{m_{k}, i_{k}}\right) \in W \backslash\{0\}
$$

where $\lambda_{1}, \ldots, \lambda_{n} \in \mathbb{C} \backslash\{0\}, m_{1}, \ldots, m_{n} \in \mathbb{N}$ and $i_{1} \in I_{m_{1}}, \ldots, i_{n} \in I_{m_{n}}$. We assume that if $j, k \in\{1, \ldots, n\}, m_{j}=m_{k}$ and $i_{j}=i_{k}$, then $j=k$. Since $\bigcup_{m=1}^{\infty} \mathcal{B}_{m}$ is a linearly independent set, $\sum_{k=1}^{n} \lambda_{k} P_{m_{k}, i_{k}} \neq 0$, so

$$
\sum_{k=1}^{n} \lambda_{k} P_{m_{k}, i_{k}} \cdot f \in \mathcal{H}(E) \backslash \mathcal{H}_{b}(E)
$$

by Proposition 3. Since $\sum_{k=1}^{n} \lambda_{k} Q_{m_{k}, i_{k}}$ is a polynomial, it is bounded on bounded subsets, so

$$
\sum_{k=1}^{n} \lambda_{k}\left(P_{m_{k}, i_{k}} \cdot f+Q_{m_{k}, i_{k}}\right) \in \mathcal{H}(E) \backslash \mathcal{H}_{b}(E) .
$$

Therefore, $W \backslash\{0\} \subset \mathcal{H}(E) \backslash \mathcal{H}_{b}(E)$.

Let us prove that $W$ is dense in $\left(\mathcal{H}(E), \tau_{\delta}\right)$. Let $h \in \mathcal{H}(E), p$ a continuous seminorm in $\left(\mathcal{H}(E), \tau_{\delta}\right)$ and $\varepsilon>0$. The Taylor series of $h$ at zero converges to $h$ for the $\tau_{\delta}$ topology (see [10, Proposition 1.2]), so there is $m \in \mathbb{N}$ such that

$$
p\left(\sum_{n=0}^{m} \frac{\widehat{d^{n}} h(0)}{n !}-h\right)<\frac{\varepsilon}{2} .
$$

If $K$ is a compact subset of $E$, then there is $t>0$ such that $t K \subset B(0,1)$. Hence

$$
\begin{aligned}
\sup _{i \in I_{m}}\left\|P_{m, i} \cdot f\right\|_{K} & \leq \frac{1}{t^{m}} \cdot \sup _{i \in I_{m}}\left\|P_{m, i}\right\|_{t K} \cdot\|f\|_{K} \\
& \leq \frac{1}{t^{m}} \cdot \sup _{i \in I_{m}}\left\|P_{m, i}\right\|_{B(0,1)} \cdot\|f\|_{K}=\frac{\|f\|_{K}}{t^{m}}<\infty .
\end{aligned}
$$

This proves that $\left\{P_{m, i} \cdot f: i \in I_{m}\right\}$ is bounded for the compact open topology, therefore for $\tau_{\delta}$ (see [10, Lemma 3.1]). Then there is $s>0$ such that

$$
s \cdot p\left(P_{m, i} \cdot f\right)<\frac{\varepsilon}{2} \quad \text { for all } i \in I_{m} .
$$

Let $i \in I_{m}$ such that

$$
Q_{m, i}=\frac{1}{s} \sum_{n=0}^{m} \frac{\widehat{d^{n}} h(0)}{n !} \in \bigoplus_{n=0}^{m} \mathcal{P}\left({ }^{n} E\right) .
$$


Then $s\left(P_{m, i} \cdot f+Q_{m, i}\right) \in W$ and

$$
\begin{aligned}
p\left(s\left(P_{m, i} \cdot f+Q_{m, i}\right)-h\right) & \leq s \cdot p\left(P_{m, i} \cdot f\right)+p\left(s Q_{m, i}-h\right) \\
& =s \cdot p\left(P_{m, i} \cdot f\right)+p\left(\sum_{n=0}^{m} \frac{\widehat{d^{n}} h(0)}{n !}-h\right)<\varepsilon .
\end{aligned}
$$

This completes the proof. The definition of $W$ is based on the proof of [7, Theorem 2.2].

Remark 6 . The density of $\mathcal{H}(E) \backslash \mathcal{H}_{b}(E)$ in $\left(\mathcal{H}(E), \tau_{\delta}\right)$ was proved by Aron in [3].

\section{Representation of the $\tau_{\delta}$ TOPOLOGY}

Let $U$ be an open subset of a Banach space $E$. If $I=\left\{V_{n}\right\}_{n=1}^{\infty}$ is an increasing countable open cover of $U$, then $\mathcal{H}_{I}(U)$ denotes the following subspace of $\mathcal{H}(U)$ :

$$
\mathcal{H}_{I}(U)=\left\{f \in \mathcal{H}(U):\|f\|_{V_{n}}<\infty \text { for all } n \in \mathbb{N}\right\},
$$

endowed with the locally convex topology defined by the seminorms

$$
f \in \mathcal{H}_{I}(U) \mapsto\|f\|_{V_{n}} \quad(n \in \mathbb{N}) .
$$

It is well known that $\left(\mathcal{H}(U), \tau_{\delta}\right)$ can be obtained as the inductive limit of the Fréchet spaces $\mathcal{H}_{I}(U)$, where $I$ ranges over the family of all increasing countable open covers of $U$ :

$$
\left(\mathcal{H}(U), \tau_{\delta}\right)=\underset{I}{\lim } \mathcal{H}_{I}(U)
$$

(see [11, Proposition 3.18]). In [1, Ansemil, Aron and Ponte showed that if $E$ is an infinite dimensional Banach space with a Schauder basis, then there is no sequence $\left\{I_{n}\right\}_{n=1}^{\infty}$ of covers of $E$, such that $\mathcal{H}(E)=\bigcup_{n=1}^{\infty} \mathcal{H}_{I_{n}}(E)$; hence the inductive limit in (3.1) is not countable. In Theorem 9 below, we will generalize this result to the case of $\left(\mathcal{H}(U), \tau_{\delta}\right)$, where $U$ will be an open subset of an arbitrary Banach space of infinite dimension. Furthermore, we will get more results on lineability of sets of holomorphic functions. With this aim we prove the following propositions.

Proposition 7. Let $U$ be an open subset of a Banach space such that $0 \in U$. Then there are sequences $\left\{z_{k}\right\}_{k=1}^{\infty} \subset U$ and $\left\{R_{k}\right\}_{k=0}^{\infty} \subset \mathbb{R}^{+}$with the following properties:

a) $\left\{R_{k}\right\}_{k=0}^{\infty}$ is an increasing sequence,

b) for each $k, z_{k} \in B\left(0, R_{k}\right)$ and $z_{k} \notin \bar{B}\left(0, R_{k-1}\right)$,

c) $U \subset \bigcup_{k=0}^{\infty} B\left(0, R_{k}\right)$.

Proof. Let $s=\sup \{\|x\|: x \in U\}$. Since $U$ is open, it is clear that $\|x\|<s$ for all $x \in U$. Let $0<R_{0}<s$. There is a vector $z_{1} \in U$ such that $R_{0}<\left\|z_{1}\right\|<s$. If $s<\infty$, then there exists $m_{1} \in \mathbb{N}$ such that

$$
\left\|z_{1}\right\|<s-\frac{1}{m_{1}}<s,
$$

and we define $R_{1}=s-\frac{1}{m_{1}}$. If $s=\infty$, we choose $R_{1} \in \mathbb{N}$ large enough so that $\left\|z_{1}\right\|<R_{1}$. In both cases we have $R_{1}<s$, so there is another vector $z_{2} \in U$ such that $R_{1}<\left\|z_{2}\right\|<s$. If $s<\infty$, then there exists $m_{2} \in \mathbb{N}, m_{2}>m_{1}$, such that

$$
\left\|z_{2}\right\|<s-\frac{1}{m_{2}}<s
$$


and we define $R_{2}=s-\frac{1}{m_{2}}$. If $s=\infty$, we choose $R_{2} \in \mathbb{N}, R_{2}>R_{1}$, large enough so that $\left\|z_{2}\right\|<R_{2}$. The sequences $\left\{z_{k}\right\}_{k=1}^{\infty}$ and $\left\{R_{k}\right\}_{k=0}^{\infty}$ are obtained by recurrence. It is clear that $z_{k} \in B\left(0, R_{k}\right), z_{k} \notin \bar{B}\left(0, R_{k-1}\right)$ for each $k$ and $\lim _{k \rightarrow \infty} R_{k}=s$. If $x \in U$, then $\|x\|<s$, so there is $k$ such that $\|x\|<R_{k}$, that is, $x \in B\left(0, R_{k}\right)$. This proves that $U \subset \bigcup_{k=0}^{\infty} B\left(0, R_{k}\right)$.

The next proposition extends a recent result by Ansemil, Aron and Ponte, who have proved that given two disjoint balls in an infinite dimensional Banach space, there is an entire function which is bounded on the first ball and unbounded on the second ball (see [2]).

Proposition 8. If $B(0, R)$ and $B\left(x_{0}, r\right)$ are disjoint balls in an infinite dimensional Banach space $E$ and $\varepsilon>0$, then there exists a sequence $\left\{f_{n}\right\}_{n=1}^{\infty}$ of entire functions on $E$ with the following properties:

a) $\left\{f_{n}\right\}_{n=1}^{\infty}$ is a linearly independent set,

b) $\left\|f_{n}\right\|_{B(0, R)}<\varepsilon$ for every $n$,

c) $\|h\|_{B\left(x_{0}, r\right)}=\infty$ for all $h \in \operatorname{span}\left\{f_{n}\right\}_{n=1}^{\infty} \backslash\{0\}$.

d) The sequence $\left\{f_{n}\right\}_{n=1}^{\infty}$ is locally bounded; that is, for every $x \in E$ there is a neighborhood $V$ of $x$ such that $\sup _{n \in \mathbb{N}}\left\|f_{n}\right\|_{V}<\infty$.

In particular, the set

$$
\left\{h \in \mathcal{H}(E):\|h\|_{B(0, R)}<\infty \text { and }\|h\|_{B\left(x_{0}, r\right)}=\infty\right\}
$$

is lineable.

Proof. By the Hahn-Banach Theorem, there exists $\varphi_{0} \in E^{\prime}$ such that $\left\|\varphi_{0}\right\|=1$ and $\varphi_{0}\left(x_{0}\right)=\left\|x_{0}\right\|$. Let $\left\{x_{k}, \varphi_{k}\right\}_{k=1}^{\infty}$ be a biorthogonal system in $E$ with the properties given in Proposition 1, Let $C>\sup _{k \in \mathbb{N}}\left\|x_{k}\right\|$.

Since $x_{0} \notin \bar{B}(0, R)$, then $\frac{R}{\left\|x_{0}\right\|}<1$, so there is $\alpha \in \mathbb{N}$ such that

$$
\left(\frac{R}{\left\|x_{0}\right\|}\right)^{\alpha}<\frac{r}{C R}
$$

that is,

$$
\frac{C \cdot R^{\alpha+1}}{\left\|x_{0}\right\|^{\alpha} \cdot r}<1 .
$$

Let $\left\{a_{n}\right\}_{n=1}^{\infty}$ be a strictly increasing sequence of positive numbers such that

$$
\frac{C \cdot R^{\alpha+1}}{\left\|x_{0}\right\|^{\alpha} \cdot r}<\cdots<\frac{1}{a_{n}}<\cdots<\frac{1}{a_{1}}<1 .
$$

This means that for all $n \in \mathbb{N}$,

$$
a_{n} \frac{C}{\left\|x_{0}\right\|^{\alpha} \cdot r} \cdot R^{\alpha+1}<1<a_{n} .
$$

For each $n \in \mathbb{N}$, the function

$$
g_{n}=\sum_{k=1}^{\infty}\left(a_{n} \frac{C}{\left\|x_{0}\right\|^{\alpha} \cdot r} \cdot \varphi_{0}^{\alpha} \cdot \varphi_{k}\right)^{k}
$$

is entire on $E$. Indeed, let $K$ be a compact subset of $E$. Since $\left\|\varphi_{k}\right\|=1$ for all $k$, the sequence $\left\{\varphi_{k}\right\}_{k=1}^{\infty}$ is equicontinuous and since $\lim _{k \rightarrow \infty} \varphi_{k}(x)=0$ for each 
$x \in E$, also $\lim _{k \rightarrow \infty}\left\|\varphi_{k}\right\|_{K}=0$ (see [14, Proposition 9.11]). Then there is $k_{0} \in \mathbb{N}$ such that

$$
a_{n} \cdot \frac{C}{\left\|x_{0}\right\|^{\alpha} \cdot r}\left\|\varphi_{0}^{\alpha}\right\|_{K} \cdot\left\|\varphi_{k}\right\|_{K} \leq \frac{1}{2}
$$

for every $k \geq k_{0}$. Thus,

$$
\sum_{k=k_{0}}^{\infty}\left(a_{n} \cdot \frac{C}{\left\|x_{0}\right\|^{\alpha} \cdot r}\left\|\varphi_{0}^{\alpha}\right\|_{K} \cdot\left\|\varphi_{k}\right\|_{K}\right)^{k} \leq \sum_{k=k_{0}}^{\infty}\left(\frac{1}{2}\right)^{k}<\infty .
$$

This implies that the series $\sum_{k=1}^{\infty}\left(a_{n} \cdot \frac{C}{\left\|x_{0}\right\|^{\alpha} \cdot r} \cdot \varphi_{0}^{\alpha} \cdot \varphi_{k}\right)^{k}$ converges uniformly on the compact subsets of $E$, so $g_{n} \in \mathcal{H}(E)$ (see [14, Proposition 9.13]). The definition of $g_{n}$ is based on the functions which appear in [1].

Let

$$
h=\sum_{n=1}^{N} \lambda_{n} g_{n} \in \operatorname{span}\left\{g_{n}\right\}_{n=1}^{\infty} \backslash\{0\},
$$

where every $\lambda_{n}$ belongs to $\mathbb{C}$ and $\lambda_{N} \neq 0$. By (3.2), each function $g_{n}$ is bounded on $B(0, R)$ :

$$
\left\|g_{n}\right\|_{B(0, R)} \leq \sum_{k=1}^{\infty}\left(a_{n} \frac{C}{\left\|x_{0}\right\|^{\alpha} \cdot r} \cdot R^{\alpha+1}\right)^{k}<\infty,
$$

so $\|h\|_{B(0, R)}<\infty$. Now let us compute the functions $g_{n}$ and $h$ at $x_{0}+\frac{r}{C} x_{k} \in$ $B\left(x_{0}, r\right)$ with $k \geq 1$ :

$$
g_{n}\left(x_{0}+\frac{r}{C} x_{k}\right)=\left(a_{n} \frac{C}{\left\|x_{0}\right\|^{\alpha} \cdot r} \cdot \varphi_{0}\left(x_{0}\right)^{\alpha} \cdot \varphi_{k}\left(\frac{r}{C} x_{k}\right)\right)^{k}=a_{n}^{k} .
$$

Therefore,

$$
h\left(x_{0}+\frac{r}{C} x_{k}\right)=\sum_{n=1}^{N} \lambda_{n} a_{n}^{k}
$$

Since $\left\{a_{n}\right\}_{n=1}^{\infty}$ is a strictly increasing sequence, $0<\frac{a_{n}}{a_{N}}<1$ for $1 \leq n \leq N-1$. Hence

$$
\frac{h\left(x_{0}+\frac{r}{C} x_{k}\right)}{a_{N}^{k}}=\lambda_{N}+\sum_{n=1}^{N-1} \lambda_{n}\left(\frac{a_{n}}{a_{N}}\right)^{k} \underset{k \rightarrow \infty}{\rightarrow} \lambda_{N} \neq 0 .
$$

As $a_{N}$ is greater than $1, \lim _{k \rightarrow \infty} a_{N}^{k}=\infty$. Therefore,

$$
\|h\|_{B\left(x_{0}, r\right)} \geq \lim _{k \rightarrow \infty}\left|h\left(x_{0}+\frac{r}{C} x_{k}\right)\right|=\infty .
$$

The sequence $\left\{g_{n}\right\}_{n=1}^{\infty}$ is linearly independent. Indeed, suppose $\sum_{n=1}^{N} \lambda_{n} g_{n}=0$, where $\left\{\lambda_{1}, \ldots, \lambda_{N}\right\} \subset \mathbb{C}$. With the same argument used to prove that $\|h\|_{B\left(x_{0}, r\right)}=$ $\infty$, we deduce that

$$
0=\frac{\sum_{n=1}^{N} \lambda_{n} g_{n}\left(x_{0}+\frac{r}{C} x_{k}\right)}{a_{N}^{k}} \underset{k \rightarrow \infty}{\rightarrow} \lambda_{N},
$$

so $\lambda_{N}=0$ and $\sum_{n=1}^{N-1} \lambda_{n} g_{n}=0$. By iterating this process, we get that $\lambda_{n}=0$ for all $n$; that is, $\left\{g_{n}\right\}_{n=1}^{\infty}$ is a linearly independent set. 
Let $K$ be a compact subset in $E$. As we have seen, $\lim _{k \rightarrow \infty}\left\|\varphi_{k}\right\|_{K}=0$, so there is $k_{0} \in \mathbb{N}$ such that

$$
\left\|\varphi_{k}\right\|_{K} \leq \frac{R^{\alpha+1}}{2\left\|\varphi_{0}\right\|_{K}^{\alpha}+1}
$$

for every $k>k_{0}$. By (3.2), we have

$$
a_{n} \frac{C}{\left\|x_{0}\right\|^{\alpha} \cdot r}<\frac{1}{R^{\alpha+1}}
$$

for every $n$, and thus we deduce the following inequalities:

$$
\begin{aligned}
\sup _{n \in \mathbb{N}}\left\|g_{n}\right\|_{K} & \leq \sum_{k=1}^{\infty}\left(\frac{1}{R^{\alpha+1}} \cdot\left\|\varphi_{0}\right\|_{K}^{\alpha} \cdot\left\|\varphi_{k}\right\|_{K}\right)^{k} \\
& \leq \sum_{k=1}^{k_{0}}\left(\frac{\left\|\varphi_{0}\right\|_{K}^{\alpha}}{R^{\alpha+1}} \cdot\left\|\varphi_{k}\right\|_{K}\right)^{k}+\sum_{k>k_{0}}\left(\frac{\left\|\varphi_{0}\right\|_{K}^{\alpha}}{R^{\alpha+1}} \cdot \frac{R^{\alpha+1}}{2\left\|\varphi_{0}\right\|_{K}^{\alpha}+1}\right)^{k} \\
& \leq \sum_{k=1}^{k_{0}}\left(\frac{\left\|\varphi_{0}\right\|_{K}^{\alpha}}{R^{\alpha+1}} \cdot\left\|\varphi_{k}\right\|_{K}\right)^{k}+1<\infty .
\end{aligned}
$$

This proves that $\left\{g_{n}\right\}_{n=1}^{\infty}$ is bounded for the compact open topology and consequently it is locally bounded (see [14, Proposition 9.15]). To conclude the proof, it suffices to define

for each $n \in \mathbb{N}$.

$$
f_{n}=\frac{\varepsilon}{\left\|g_{n}\right\|_{B(0, R)}+1} g_{n}
$$

Theorem 9. Let $U$ be an open subset of an infinite dimensional Banach space E. For each $k \in \mathbb{N}$, let $I_{k}=\left\{V_{k, j}\right\}_{j=1}^{\infty}$ denote an increasing countable open cover of $U$. Then there exists an infinite dimensional vector space $W$ such that

$$
W \backslash\{0\} \subset \mathcal{H}(U) \backslash \bigcup_{k=1}^{\infty} \mathcal{H}_{I_{k}}(U) .
$$

In particular, $\mathcal{H}(U) \neq \bigcup_{k=1}^{\infty} \mathcal{H}_{I_{k}}(U)$, so the inductive limit in (3.1) is not countable.

Proof. Some of our arguments are based on the proof of the main theorem in 1. Using a translation, we can assume that $0 \in U$. By Proposition 7 , there are sequences $\left\{z_{k}\right\}_{k=1}^{\infty} \subset U$ and $\left\{R_{k}\right\}_{k=0}^{\infty} \subset \mathbb{R}^{+}$such that $\left\{R_{k}\right\}_{k=0}^{\infty}$ is increasing, $U \subset \bigcup_{k=0}^{\infty} B\left(0, R_{k}\right), z_{k} \in B\left(0, R_{k}\right)$ and $z_{k} \notin \bar{B}\left(0, R_{k-1}\right)$ for every $k \geq 1$.

The collection $I_{1}=\left\{V_{1, j}\right\}_{j=1}^{\infty}$ is an open cover of $U$, so there is $j_{1} \in \mathbb{N}$ such that $z_{1} \in V_{1, j_{1}}$. Then there exists $r_{1}>0$ such that

$$
B\left(z_{1}, r_{1}\right) \subset V_{1, j_{1}}, \quad B\left(z_{1}, r_{1}\right) \subset B\left(0, R_{1}\right) \quad \text { and } \quad B\left(z_{1}, r_{1}\right) \cap B\left(0, R_{0}\right)=\emptyset .
$$

By Proposition 8 , there exists a linearly independent sequence $\left\{f_{1, n}\right\}_{n=1}^{\infty} \subset \mathcal{H}(E)$ such that

$$
\left\|f_{1, n}\right\|_{B\left(0, R_{0}\right)}<\frac{1}{2} \quad \text { for each } n
$$

and

$$
\|h\|_{B\left(z_{1}, r_{1}\right)}=\infty \quad \text { for all } h \in \operatorname{span}\left\{f_{1, n}\right\}_{n=1}^{\infty} \backslash\{0\}
$$


$I_{2}=\left\{V_{2, j}\right\}_{j=1}^{\infty}$ is also an open cover of $U$, so there is $j_{2} \in \mathbb{N}$ such that $z_{2} \in V_{2, j_{2}}$. Since the sequence $\left\{f_{1, n}\right\}_{n=1}^{\infty}$ is locally bounded, there exists $r_{2}>0$ such that

$$
B\left(z_{2}, r_{2}\right) \subset V_{2, j_{2}}, \quad B\left(z_{2}, r_{2}\right) \subset B\left(0, R_{2}\right), \quad B\left(z_{2}, r_{2}\right) \cap B\left(0, R_{1}\right)=\emptyset
$$

and

$$
\left\|f_{1, n}\right\|_{B\left(z_{2}, r_{2}\right)}<\infty \quad \text { for all } n .
$$

Again, there exists a linearly independent sequence $\left\{f_{2, n}\right\}_{n=1}^{\infty} \subset \mathcal{H}(E)$ such that

$$
\left\|f_{2, n}\right\|_{B\left(0, R_{1}\right)}<\frac{1}{2^{2}} \quad \text { for each } n
$$

and

$$
\|h\|_{B\left(z_{2}, r_{2}\right)}=\infty \quad \text { for all } h \in \operatorname{span}\left\{f_{2, n}\right\}_{n=1}^{\infty} \backslash\{0\} .
$$

By iterating this process, we get sequences $\left\{j_{k}\right\}_{k=1}^{\infty} \subset \mathbb{N},\left\{r_{k}\right\}_{k=1}^{\infty} \subset \mathbb{R}^{+}$and $\left\{f_{k, n}\right\}_{k, n=1}^{\infty} \subset \mathcal{H}(E)$ with the following properties:
a) $B\left(z_{k}, r_{k}\right) \subset V_{k, j_{k}}$,
b) $B\left(z_{k}, r_{k}\right) \subset B\left(0, R_{k}\right)$,
c) $B\left(z_{k}, r_{k}\right) \cap B\left(0, R_{k-1}\right)=\emptyset$,
d) $\left\|f_{j, n}\right\|_{B\left(z_{k}, r_{k}\right)}<\infty$ if $j<k$ and $n \in \mathbb{N}$,
e) $\left\|f_{k, n}\right\|_{B\left(0, R_{k-1}\right)}<\frac{1}{2^{k}}$ for all $n$,
f) $\|h\|_{B\left(z_{k}, r_{k}\right)}=\infty$ for all $h \in \operatorname{span}\left\{f_{k, n}\right\}_{n=1}^{\infty} \backslash\{0\}$.

Let $n \in \mathbb{N}$. If $K$ is a compact subset of $U$, then there is $k \in \mathbb{N}$ such that $K \subset B\left(0, R_{k}\right)$ and thus

$$
\sum_{j=k+1}^{\infty}\left\|f_{j, n}\right\|_{K} \leq \sum_{j=k+1}^{\infty}\left\|f_{j, n}\right\|_{B\left(0, R_{k}\right)} \leq \sum_{j=k+1}^{\infty}\left\|f_{j, n}\right\|_{B\left(0, R_{j-1}\right)} \leq \sum_{j=k+1}^{\infty} \frac{1}{2^{j}}<\infty .
$$

Therefore, the series $\sum_{j=1}^{\infty} f_{j, n}$ converges uniformly on the compact subsets of $U$. Since each $f_{j, n}$ is entire, the function

$$
g_{n}=\sum_{j=1}^{\infty} f_{j, n}
$$

is holomorphic on $U$ (see [14, Proposition 9.13]). Let $W$ be the vector space generated by $\left\{g_{n}\right\}_{n=1}^{\infty}$.

If $\lambda_{1}, \ldots, \lambda_{n} \in \mathbb{C}$ and $\lambda_{1} g_{1}+\cdots+\lambda_{n} g_{n}=0$, then

$$
\begin{aligned}
0= & \left\|\lambda_{1} g_{1}+\cdots+\lambda_{n} g_{n}\right\|_{B\left(z_{1}, r_{1}\right)} \\
\geq & \left\|\lambda_{1} f_{1,1}+\cdots+\lambda_{n} f_{1, n}\right\|_{B\left(z_{1}, r_{1}\right)} \\
& -\sum_{j=2}^{\infty}\left(\left\|\lambda_{1} f_{j, 1}\right\|_{B\left(z_{1}, r_{1}\right)}+\cdots+\left\|\lambda_{n} f_{j, n}\right\|_{B\left(z_{1}, r_{1}\right)}\right) \\
\geq & \left\|\lambda_{1} f_{1,1}+\cdots+\lambda_{n} f_{1, n}\right\|_{B\left(z_{1}, r_{1}\right)} \\
& -\sum_{j=2}^{\infty}\left(\left\|\lambda_{1} f_{j, 1}\right\|_{B\left(0, R_{j-1}\right)}+\cdots+\left\|\lambda_{n} f_{j, n}\right\|_{B\left(0, R_{j-1}\right)}\right) .
\end{aligned}
$$


We now use property (e):

$$
0 \geq\left\|\lambda_{1} f_{1,1}+\cdots+\lambda_{n} f_{1, n}\right\|_{B\left(z_{1}, r_{1}\right)}-\sum_{j=2}^{\infty}\left(\frac{\left|\lambda_{1}\right|}{2^{j}}+\cdots+\frac{\left|\lambda_{n}\right|}{2^{j}}\right)
$$

This implies that

$$
\left\|\lambda_{1} f_{1,1}+\cdots+\lambda_{n} f_{1, n}\right\|_{B\left(z_{1}, r_{1}\right)} \leq\left(\left|\lambda_{1}\right|+\cdots+\left|\lambda_{n}\right|\right) \sum_{j=2}^{\infty} \frac{1}{2^{j}}<\infty .
$$

By (f) we deduce that $\lambda_{1} f_{1,1}+\cdots+\lambda_{n} f_{1, n}=0$. Then $\lambda_{1}=\cdots=\lambda_{n}=0$, since $f_{1,1}, \ldots, f_{1, n}$ are linearly independent. This shows that the functions $\left\{g_{n}\right\}_{n=1}^{\infty}$ are also linearly independent and that $W$ has infinite dimension.

Now we are going to prove that $W \backslash\{0\} \subset \mathcal{H}(U) \backslash \bigcup_{k=1}^{\infty} \mathcal{H}_{I_{k}}(U)$. Let $h=$ $\lambda_{1} g_{1}+\cdots+\lambda_{n} g_{n} \in W$, where at least $\lambda_{n} \neq 0$. Let $k \in \mathbb{N}$. Then

$$
\begin{aligned}
\|h\|_{V_{k, j_{k}}} \geq & \|h\|_{B\left(z_{k}, r_{k}\right)} \\
\geq & \left\|\lambda_{1} f_{k, 1}+\cdots+\lambda_{n} f_{k, n}\right\|_{B\left(z_{k}, r_{k}\right)} \\
& -\sum_{j=1}^{k-1}\left(\left\|\lambda_{1} f_{j, 1}\right\|_{B\left(z_{k}, r_{k}\right)}+\cdots+\left\|\lambda_{n} f_{j, n}\right\|_{B\left(z_{k}, r_{k}\right)}\right) \\
& -\sum_{j=k+1}^{\infty}\left(\left\|\lambda_{1} f_{j, 1}\right\|_{B\left(z_{k}, r_{k}\right)}+\cdots+\left\|\lambda_{1} f_{j, n}\right\|_{B\left(z_{k}, r_{k}\right)}\right) .
\end{aligned}
$$

If $j \geq k+1$, then $B\left(z_{k}, r_{k}\right) \subset B\left(0, R_{j-1}\right)$ and by (e),

$$
\begin{aligned}
\|h\|_{V_{k, j_{k}}} \geq & \left\|\lambda_{1} f_{k, 1}+\cdots+\lambda_{n} f_{k, n}\right\|_{B\left(z_{k}, r_{k}\right)} \\
& -\sum_{j=1}^{k-1}\left(\left\|\lambda_{1} f_{j, 1}\right\|_{B\left(z_{k}, r_{k}\right)}+\cdots+\left\|\lambda_{n} f_{j, n}\right\|_{B\left(z_{k}, r_{k}\right)}\right) \\
& -\left(\left|\lambda_{1}\right|+\cdots+\left|\lambda_{n}\right|\right) \sum_{j=k+1}^{\infty} \frac{1}{2^{j}} .
\end{aligned}
$$

Since $f_{k, 1}, \ldots, f_{k, n}$ are linearly independent, $\lambda_{1} f_{k, 1}+\cdots+\lambda_{n} f_{k, n} \neq 0$. By (f),

$$
\left\|\lambda_{1} f_{k, 1}+\cdots+\lambda_{n} f_{k, n}\right\|_{B\left(z_{k}, r_{k}\right)}=\infty .
$$

By (d), if $1 \leq j \leq k-1$, then

$$
\left\|f_{j, 1}\right\|_{B\left(z_{k}, r_{k}\right)}<\infty, \ldots,\left\|f_{j, n}\right\|_{B\left(z_{k}, r_{k}\right)}<\infty .
$$

Hence $\|h\|_{V_{k, j_{k}}}=\infty$ and $h$ does not belong to any of the spaces $\mathcal{H}_{I_{k}}(U)$. This completes the proof.

\section{ACKNOWLEDGMENTS}

The author would like to thank Professors Ansemil and Ponte for their help and suggestions during the preparation of this article. 


\section{REFERENCES}

1. J. M. Ansemil, R. M. Aron, S. Ponte, Representation of spaces of entire functions on Banach spaces, Publ. Res. Inst. Math. Sci., 45 (2009), 383-391. MR2510506 (2010c:46105)

2. J. M. Ansemil, R. Aron, S. Ponte, Behavior of entire functions on balls in a Banach space, to appear in Indag. Math.

3. R. M. Aron, Entire functions of unbounded type on a Banach space, Boll. Unione Mat. Ital., 9 (1974), 28-31. MR0374911 (51:11107)

4. R. M. Aron, M. Schottenloher, Compact holomorphic mappings on Banach spaces and the approximation property, J. Funct. Anal., 21 (1976), 7-30. MR0402504 (53:6323)

5. R. M. Aron, V. I. Gurariy, J. B. Seoane Sepúlveda, Lineability and spaceability of sets of functions on $\mathbb{R}$, Proc. Amer. Math. Soc., 133 (2005), 795-803. MR 2113929 (2006i:26004)

6. R. M. Aron, J. B. Seoane Sepúlveda, Algebrability of the set of everywhere surjective functions on $\mathbb{C}$, Bull. Belg. Math. Soc. Simon Stevin, 14 (2007), 25-31. MR2327324 (2008d:26016)

7. R. M. Aron, F. J. García Pacheco, D. Pérez García, J. B. Seoane Sepúlveda, On denselineability of sets of functions on $\mathbb{R}$, Topology, 48 (2009), 149-156. MR2596209

8. C. Benítez, Y. Sarantopoulos, A. Tonge, Lower bounds for norms of products of polynomials, Math. Proc. Cambridge Philos. Soc., 124 (1998), pp. 395-408. MR.1636556 (99h:46077)

9. S. J. Dilworth, M. Girardi, W. B. Johnson, Geometry of Banach spaces and biorthogonal systems, Studia Math., 140 (2000), 243-271. MR.1784153 (2001i:46013)

10. S. Dineen, Holomorphic functions on locally convex topological vector spaces. I, Locally convex topologies on $\mathcal{H}(U)$, Ann. Inst. Fourier (Grenoble), 23 (1973), 19-54. MR0500153 (58:17843)

11. S. Dineen, Complex Analysis on Infinite Dimensional Spaces, Springer Monogr. Math., 1999. MR 1705327 (2001a:46043)

12. V. I. Gurariǔ, Subspaces and bases in spaces of continuous functions, Dokl. Akad. Nauk SSSR, 167 (1966), 971-973. MR0199674 (33:7817)

13. L. Halbeisen, N. Hungerbühler, The cardinality of Hamel bases of Banach spaces, East-West J. Math., 2 (2000), 153-159. MR.1825451 (2002f:46013)

14. J. Mujica, Complex Analysis in Banach Spaces, North-Holland Math. Stud., 120, Amsterdam, 1986. MR842435 (88d:46084)

Departamento de Análisis Matemático, Universidad Complutense de Madrid, 28040 MADRID, SPAIN

E-mail address: jlopezsalazar@mat.ucm.es 